\begin{tabular}{|c|l|}
\hline Title & External electric field effects on fluorescence of perylene doped in a polymer film \\
\hline Author(s) & A ra, A njue Mane; limori, Toshifumi; Y oshizawa, Tomokazu; Nakabay ashi, Takakazu; Ohta, Nobuhiro \\
\hline Citation & $\begin{array}{l}\text { Chemical Physics Letters, 427(46), 322-328 } \\
\text { https://doi.org/10.1016/.cplett.2006.07.001 }\end{array}$ \\
\hline Issue Date & 2006 \\
\hline Doc URL & http://hdl.handle.net/2115/14741 \\
\hline Type & article (author version) \\
\hline File Information & CPL427-46.pdf \\
\hline
\end{tabular}

Instructions for use 


\section{External electric field effects on fluorescence of perylene doped in a polymer film}

Anjue Mane Ara, ${ }^{1}$ Toshifumi Iimori, ${ }^{1,}{ }^{2}$ Tomokazu Yoshizawa, ${ }^{1}$ Takakazu Nakabayashi, ${ }^{1,}{ }^{2}$ and Nobuhiro Ohta ${ }^{1,2}$

${ }^{1}$ Graduate School of Environmental Earth Science, Hokkaido University, Sapporo 060-0812, Japan.

${ }^{2}$ Research Institute for Electronic Science, Hokkaido University, Sapporo 060-0812, Japan.

Abstract:

Fluorerescence and electrofluorescence spectra of perylene molecules doped in a polymer film at different concentrations have been measured at different temperatures in the range of 70-295 K. At high concentrations where excimer is formed, LE fluorescence emitted from the locally excited state of perylene is quenched by an electric field at any temperature, indicating a field-induced enhancement of the excimer formation rate. Fluorescence of the Y-type excimer of perylene molecules shows a fieldinduced enhancement at room temperature, whereas it shows a field-induced quenching at low temperatures. The temperature dependence of the electric field effects on fluorescence has been discussed.

* Corresponding author. E-mail: nohta@es.hokudai.ac.jp 


\section{Introduction}

Perylene molecules are widely used as a fluorescence probe in different research fields since it forms a characteristic excimer in the excited state. High fluorescence quantum yield, photostability, thermal stability and efficient carrier mobility also make perylene an excellent candidate as organic semiconductor as well as organic electroluminescence (EL) material [1-3]. Owing to the highly hydrophobic property and red color emission of excimer, perylene molecules have been used in several biological investigations such as DNA detection and probing of the physical states of membrane [4-6].

Measurements of electric field effects on optical spectra are very useful to examine both electronic properties in excited states and electric field effects on photoexcitation dynamics. It is well established that electric field effects on molecular complexes such as exciplex or excimer are informative to elucidate the excited state dynamics in mixtures of electron donor and acceptor compounds or in chainlinked systems where two different or same reactants are attached to chain ends [7-10]. Such studies provide the pathways to develop new materials having photofunctions such as photoconductivity or EL property, which provides a favorable outlook for optoelectronics applications.

Excimer formation processes of perylene molecules have been studied for a long time under various conditions; in solution [11], in crystalline[12,13], in thin polymer film [14], in polymer matrix [15], in LB film [16,17] and in rigid media [18]. It is well known that perylene excimer shows a characteristic feature at low temperatures $[12,13,15,16]$. As shown in pyrene [19], a combination of electric field effect and temperature effect on fluorescence is very useful to understand the excited state dynamic of perylene complexes. However, the electric field effects on excitation dynamics have not been examined for perylene molecules. In the present study, we have measured fluorescence and electrofluorescence spectra of perylene molecules doped in a poly (methyl methacrylate) (PMMA) polymer film at different concentrations at different temperatures. Based on the results, excitation dynamics as well as its electric field effect has been discussed for perylene doped in a PMMA film. 


\section{Experimental}

Perylene purified by a sublimation (Sigma Aldrich) was used without further purification. PMMA (Aldrich, MW 120000) was purified by precipitation with methanol and benzene. The concentrations of perylene relative to the monomer unit of PMMA employed in the present study were $0.1,1.0$ and 3.0 mol\%. Spectroscopic grade chloroform (Kanto chemicals) was used as a solvent.

Sample preparation and experimental apparatus used in the present experiments are essentially the same with the ones reported in our previous paper [19]. A quartz plate which is free from impurity emission was used as a substrate. A certain amount of chloroform solution of perylene and PMMA was cast onto an ITO-coated quartz substrate by a spin coating method. The sample was dried slowly in the dark at room temperature and then dried under vacuum condition. Then, a semitransparent aluminum (Al) film was deposited on the dried polymer film by using a vacuum vapor deposition method. The ITO and Al films were used as electrodes for applying electric fields. Hereafter, applied electric field is denoted by $\boldsymbol{F}$ and its strength is represented by rms. The thickness of the polymer film, which was typically $0.5 \mu \mathrm{m}$, was determined using a thickness measurement system (NanoSpec/AFT model 010-0180, Nanometrics Inc.). The strength of the external electric fields was calculated from the applied voltage divided by the film thickness.

In the low temperature measurements, the same experimental apparatus with the one described elsewhere was used [19]. Briefly, the sample was mounted on a holder and cooled using a cryogenic refrigerating system (Diakin, V202C5LR) equipped with quartz optical windows, and the temperature (T) of the substrate was monitored using a temperature controller (Scientific Instruments, model 9600) with a silicon diode thermometer (Scientific Instruments, Si410A). The substrate was excited from the semitransparent $\mathrm{Al}$ side with an incident angle of $\sim 45^{\circ}$, and undesirable detection of the scattered light of the excitation beam was effectively avoided. All the optical spectra were measured under vacuum conditions. Electric-field-induced changes in absorption and emission spectra were measured using 
electric field modulation spectroscopy with the same apparatus as described elsewhere [8-10]. A sinusoidal ac voltage was applied to a sample with a modulation frequency of $40 \mathrm{~Hz}$. Field-induced change in transmitted excitation light intensity or emission intensity was detected with a lock-in amplifier at the second harmonic of the modulation frequency, and the electroabsorption or electrofluorescence spectra were obtained as a function of wavelength. Fluorescence spectra were simultaneously observed by monitoring the dc component of the fluorescence intensity.

\section{Results and discussion}

Electroabsorption spectra of perylene show only the Stark shift given by the first derivative of the absorption spectrum at every concentration and at any temperature, indicating that the temperature effect on absorption spectra as well as on electroabsorption spectra of perylene is not significant.

Electrofluorescence spectra as well as fluorescence spectra of perylene in a PMMA film at a low concentration of 0.1 mol\% taken at $T=295 \mathrm{~K}$ and $70 \mathrm{~K}$ are shown in Figure 1 . Hereafter, electrofluorescence spectrum, i.e., plots of the field-induced change in fluorescence intensity as a function of wavlength/wavenumber, is denoted by E-F spectrum. Note that the excitation wavelength where the field-induced change in absorption intensity was negligible was always used to obtain E-F spectra in the present study. The fluorescence spectrum shows well defined vibronic bands and is assigned as the LE fluorescence emitted from the locally excited state of perylene. The shape of the EF spectrum is essentially the same as the first derivative of the fluorescence spectrum irrespective of temperatures (see Figure 1), indicating that the field-induced change in fluorescence intensity essentially comes from the change in molecular polarizability following emission at $0.1 \mathrm{~mol} \%$.

As the concentration increases, LE fluorescence becomes weaker, while a broad fluorescence with a peak at $515 \mathrm{~nm}\left(\sim 19420 \mathrm{~cm}^{-1}\right)$ becomes stronger. This broad emission is assigned as the excimer florescence of perylene. Figure 2 shows E-F spectra as well as fluorescence spectra of perylene doped 
in a PMMA film observed at an intermediate concentration of $1.0 \mathrm{~mol} \%$ at different temperatures. At $T$ $=295 \mathrm{~K}$, the E-F spectrum in the region of the LE fluorescence is similar in shape to the LE fluorescence spectrum, indicating that the quantum yield of the LE fluorescence decreases in the presence of an electric field. Actually, the E-F spectrum of the LE fluorescence is simulated by a linear combination between the LE fluorescence spectrum and its first derivative spectrum; both field-induced quenching and Stark shift are observed for the LE fluorescence. The E-F spectra of excimer fluorescence are similar in shape to the first derivative of the excimer fluorescence spectrum, implying the presence of the Stark shift. Further, the intensity of the E-F spectrum integrated over the spectral region of the excimer fluorescence is positive at room temperature, indicating that the excimer fluorescence increases in the presence of an electric field. At a low temperature of $70 \mathrm{~K}$, on the other hand, the intensity of the E-F spectrum integrated in the region of the excimer fluorescence is negative, indicating that the excimer fluorescence decreases in the presence of an electric field at low temperatures.

At a high concentration of 3.0 mol \%, fluorescence spectra are dominated by the broad excimer fluorescence with a peak at $530 \mathrm{~nm}\left(\sim 18870 \mathrm{~cm}^{-1}\right)$ irrespective of temperature (Figure 3). Because of the weakness of the LE fluorescence at this high concentration, the E-F spectrum of the excimer can be clearly extracted. As in the case at $1.0 \mathrm{~mol} \%$, E-F spectra observed at $3.0 \mathrm{~mol} \%$ show a very different shape from the excimer fluorescence spectra. Actually, the E-F spectra at $3.0 \mathrm{~mol} \%$ could be simulated by a linear combination of the fluorescence spectrum (denoted as $I_{F}(\bar{v})$ ) and its first derivative spectrum as follows:

$$
\Delta I_{\mathrm{F}}(\bar{v})=a I_{F}(\bar{v})+b\left(\mathrm{~d} I_{\mathrm{F}}(\bar{v}) / \mathrm{d} \bar{v}\right)
$$

where $\bar{v}$ denotes wavenumber, and $a$ and $b$ are coefficients. The results of the simulation using equation (1) at 295 and $70 \mathrm{~K}$ are shown in Figure 4, and the following coefficients were obtained: $a$ $=5.3 \times 10^{-3}$ and $b=9.5$ at $T=295 \mathrm{~K}$, and $a=-2.5 \times 10^{-3}$ and $b=7.0$ at $T=70 \mathrm{~K}$ with a field strength of 
1.0 $\mathrm{MVcm}^{-1}$. Note that $a$ is a positive value at room temperature while it is a negative value at $T=70$ $\mathrm{K}$, which confirms the field-induced enhancement at $295 \mathrm{~K}$ and the field-induced quenching at $70 \mathrm{~K}$. Note that the zeroth derivative component corresponds to the change in the fluorescence quantum yield and that the first derivative component corresponds to the Stark shift due to the change in molecular polarizability between the excited state and the ground state.

Plots both of the intensity $\left(I_{\mathrm{F}}\right)$ and of its field-induced change $\left(\Delta I_{\mathrm{F}}\right)$ relative to $I_{\mathrm{F}}$ as a function of temperature were obtained. The results are shown in Figure 5 for both LE fluorescence and excimer fluorescence at $1.0 \mathrm{~mol} \%$ and for excimer fluorescence at $3.0 \mathrm{~mol} \%$. LE fluorescence at $448 \mathrm{~nm}$ and excimer fluorescence at $515 \mathrm{~nm}$ were monitored in Figures 5a and 5b, and excimer fluorescence at 530 nm was monitored in Figure 5c. Note that there is no contribution of the first derivative component at these monitoring wavelengths, where fluorescence shows a peak. As shown in Figure 5a, LE fluorescence at $1.0 \mathrm{~mol} \%$ shows a field-induced quenching at $295 \mathrm{~K}$, and the magnitude of the quenching relative to the total intensity of the LE fluorescence, i.e., $\Delta I_{\mathrm{F}} / I_{\mathrm{F}}$, is roughly constant at different temperatures. It is noted that the LE fluorescence once becomes weaker and then becomes stronger below $150 \mathrm{~K}$, as the temperature decreases from $295 \mathrm{~K}$. At $1.0 \mathrm{~mol} \%$, the intensity of the excimer fluorescence monotonically increases, as the temperature decreases. A field-induced enhancement is observed for excimer fluorescence at $295 \mathrm{~K}$, but excimer fluorescence as well as LE fluorescence is quenched in the presence of an electric field at low temperatures. The magnitude of the quenching of the exciemr fluorescence, i.e., $\left|\Delta I_{\mathrm{F}} / I_{\mathrm{F}}\right|$, becomes larger, as the temperature decreases (see Figure 5b). As shown in Figure 5c, similar temperature dependence was observed for excimer fluorescence at $3.0 \mathrm{~mol} \%$, i. e., field-induced enhancement at $T=295 \mathrm{~K}$ and field-induced quenching at low temperatures. From these plots, two features are noted for the excimer fluorescence. One is the opposite field-dependence at $T=295 \mathrm{~K}$ and at low temperatures. Another is the monotonic increase of the field-induced quenching with a decrease of temperature. It should be also noted that both LE 
fluorescence and excimer fluorescence show quadratic field dependence irrespective of temperature at any concentration.

It is well known that perylene molecules form two types of excimer [12]: 1) E-type excimer which has a sandwich conformation; 2) Y-type excimer which has a partially overlapping conformation. In $\alpha$-perylene crystal, the fluorescence peak of E-type excimer is at around $630 \mathrm{~nm}$, while the fluorescence peak of Y-type excimer is at around $530 \mathrm{~nm}$ [12]. In L-B films [16,17], these peaks are slightly shifted, but the fluorescence peak of Y-type excimer is consistent with the one of the excimer observed in the present study. Thus, the present results suggest that perylene molecules doped in a PMMA film at high concentrations exhibit Y-type excimer and that the E-type excimer is negligible, though configurational coordinates of the Y-type excimer may be subject to some statistical spread [15].

Field-induced quenching of the LE fluorescence of perylene doped in a PMMA film observed only at high concentrations may be attributed to a field-induced enhancement of the excimer formation rate. As a result of the field-induced enhancement of the excimer formation rate, the quantum yield of the excimer may be increased in the presence of an electric field. Y-type excimer of peryrene may be also produced through a contact pair of perylene, not through the LE fluorescent state, and the field-induced enhancement of this process may also induce an increase of the excimer concentration, as in the case of the second excimer of pyrene [20]. Thus, the field-induced increase of the intensity of the excimer fluorescence at high temperatures may be attributed to the field-induced increase in concentration of excimer. On the other hand, the field-induced quenching of the excimer fluorescence observed at low temperatures can be attributed to a field-induced acceleration of the nonradiative process of the fluorescent excimer and/or to a field-assisted dissociation of the excimer. The excimer may have a charge-separated character, and so a hole-electron pair which can be dissociated by an electric field may be produced in a polymer film.

There is no doubt that the field-induced quenching of the excimer fluorescence of perylene doped in a PMMA film becomes larger, as the temperature decreases (see Figure 5). This temperature 
dependence of the field-induced quenching of the excimer fluorescence reminds us the temperature dependence of the electron carrier mobility in $\alpha$-perylene crystal. The temperature dependent carrier mobility of this crystal has been described over a wide temperature range (40 - $300 \mathrm{~K}$ ) by a typical power law [21,22]: $\mu(T) \propto T^{-n}$, where $\mu$ is the carrier mobility, and $n$ is ranged from 1.72 to 2.15 , depending on the crystal direction, i.e., $\mu(T)$ increases with a decrease of temperature in crystal. It was shown that the field-induced quenching of exciplex or excimer fluorescence is deeply related to the transport properties $[8,10]$. As the temperature decreases, the electron carrier mobility increases and accordingly the field-induced dissociation of the excimer may be more significant. If microcrystals of perylene are formed in a polymer film, the field-induced quenching of excimer fluorescence becomes larger, as the temperature decreases. At high temperatures, on the other hand, the field-induced increase of the excimer concenration may be dominant. This leads to the switching of the field-induced effect on the excimer fluorescence of perylene; field-induced enhancement at high temperatures and field-induced quenching at low temperatures. The absorption spectra observed in the present experiments are similar to the one in solution even at the highest concentration of $3 \mathrm{~mol} \%$ and very different from the ones reported for perylene microcrystals whose size are more than $50 \mathrm{~nm}$ [23]. If the observed temperature dependence of the field-induced change in excimer fluorescence results from the microcrystal formation, the diameter of perylene microcrystals formed in a PMMA film must be much smaller than $50 \mathrm{~nm}$. With respect to the presence of the microcrystals smaller than $50 \mathrm{~nm}$, further study is necessary. It is generally considered that the carrier mobility in amorphous polymer like polyvinyl carbazole or doped polymers increases with temperature because the carrier hopping can be assisted with the thermal motion of polymer chains. Thus, the observed temperature dependence of the field-induced quenching of the excimer fluorescence seems to be inconsistent with the temperature dependence of the conductivity of doped polymers. However, it may be also necessary to examine the 
possibility that perylene molecules doped in a PMMA film at high concentrations give a conductivity similar to the one expected for crystals because of the crystal-like network formation in PMMA.

Besides the field-induced change in excited state dynamics, the so-called Stark shift is induced in the fluorescence spectra by an electric field, depending on the magnitude of electric dipole moment and molecular polarizability in the ground and excited states. As a result, fluorescence spectra are influenced by an electric field without any change in emission quantum yield. In rigid matrices such as PMMA films, the effect of the field-induced orientation of the ground-state or the excited-state molecules of perylene is assumed to be negligible since perylene has an inversion symmetry. With these assumptions and with the assumption of isotropic molecular properties, $\Delta I_{\mathrm{F}}$ induced by a change in electric dipole moment and molecular polarizability can be expressed in terms of the field-free emission intensity as [24]

$$
\begin{aligned}
\Delta I_{F}= & \left\{\left[\Delta \bar{\alpha} / 2+\left(\Delta \alpha_{m}-\Delta \bar{\alpha}\right)\left(3 \cos ^{2} \chi-1\right) / 10\right](h c)^{-1} \bar{v}^{3}\left[d\left(I_{F} / \bar{v}^{3}\right) / d \bar{v}\right]\right. \\
& \left.+(\Delta \mu)^{2}\left[5+\left(3 \cos ^{2} \chi-1\right) \times\left(3 \cos ^{2} \xi-1\right)\right] 30^{-1}(h c)^{-2} \bar{v}^{3}\left[d^{2}\left(I_{F} / \bar{v}^{3}\right) / d \bar{v}^{2}\right]\right\}|f \boldsymbol{F}|^{2}
\end{aligned}
$$

Here, $h$ represents the Planck's constant, $\Delta \mu$ and $\Delta \alpha$ are the differences in electric dipole moment and molecular polarizability, respectively, between the ground state (g) and the emitting state (e), i.e., $\Delta \boldsymbol{\mu}=$ $\mu_{\mathrm{e}}-\mu_{\mathrm{g}}$, and $\Delta \boldsymbol{\alpha}=\boldsymbol{\alpha}_{\mathrm{e}}-\boldsymbol{\alpha}_{\mathrm{g}}$. They are given as follows: $\Delta \mu=|\Delta \mu| ; \Delta \bar{\alpha}=(1 / 3) \operatorname{Tr}(\Delta \alpha) . \quad \chi$ is the angle between the direction of $\boldsymbol{F}$ and the electric vector of the excitation light, $\xi$ is the angle between the direction of $\Delta \boldsymbol{\mu}$ and the transition dipole moment, $\Delta \alpha_{m}$ denotes the diagonal component of $\Delta \boldsymbol{\alpha}$ with respect to the direction of the transition dipole moment, and $f$ is the internal field factor.

As already mentioned, the first derivative component of the excimer fluorescence spectrum is important for simulation of the E-F spectra both at room temperature and at low temperatures, and the term which corresponds to the second derivative is not necessary (see equation (1) and Figure 4). 
These results indicate that $\Delta \boldsymbol{\mu}$ of the excimer is negligible. If the polarizability is assumed to be isotropic, i.e., $\Delta \alpha_{m}=\Delta \bar{\alpha}$, equation (3) becomes

$$
\Delta I_{F}=[\Delta \bar{\alpha} / 2](h c)^{-1} \bar{v}^{3}\left[d\left(I_{F} / \bar{v}^{3}\right) / d \bar{v}\right]|\boldsymbol{F}|^{2}
$$

By assuming that $b$ corresponds to $[\Delta \bar{\alpha} / 2](h c)^{-1}$ (see equation (1)), $\Delta \alpha$ of Y-type excimer is then estimated to be 340 in units of $\AA^{3}$ at $T=295 \mathrm{~K}$, and this value becomes a little smaller at $T=70 \mathrm{~K}$, i.e., $250 \AA^{3}$. Here, it is assumed that $f=1$. LE fluorescence of perylene also shows the Stark shift, which results from the difference in molecular polarizability between the LE state and the ground state. The magnitude of $\Delta \alpha$ is also estimated from the E-F spectra at $0.1 \mathrm{~mol} \%$ to be $\backsim 70 \quad \AA^{3}$ both at $295 \mathrm{~K}$ and at $70 \mathrm{~K}$, which is much smaller than the $\Delta \alpha$ values of the excimer.

\section{Acknowledgements}

This work has been supported by Grants-in-Aid for Scientific Research (Grant No. 15205001) and for Scientific Research on Priority Area “Molecular Nano Dynamics” from the Ministry of Education, Culture, Sports, Science, and Technology in Japan. 


\section{References}

[1] S. H. Kim, Y. S. Yang, J. H. Lee, J.-I. Lee, H. Y. Chu, H. Lee, J. Oh, L.-M. Do and T. Zyung, Opt. Mat. 21 (2002) 439.

[2] S. Toguchi, Y. Morioka, H. Ishikawa, A. Oda and E. Hasegawa, Synth. Met. 111 (2000) 57.

[3] Y. Toda and H. Yanagi, Appl. Phys. Lett. 69 (1996) 2315.

[4] H. Schott, D. Von Cunow and H. Langhals, Biochimica et Biophysica Acta. 1110 (1992) 151.

[5] R. E. Pagano, K. Ozato and J.-M. Ruysschaert, Biochimica et Biophysica Acta. 465 (1997) 661.

[6] L. H. Hurley, R. T. Wheelhouse, D. Sun, S. M. Kerwin, M. Salazar, O. Y. Fedoroff, F. X. Han, H. Han, E. Izbicka and D. D. Von Hoff, Pharmacology \& Therapeutics. 85 (2000) 141.

[7] M. Yokoyama, Y. Endo and H. Mikawa, Bull. Chem. Soc. Jpn. 49 (1976) 1538.

[8] N. Ohta, S. Umeuchi, Y. Nishimura and I. Yamazaki J. Phys. Chem. B 102 (1998) 3784.

[9] N. Ohta, M. Koizumi, Y. Nishimura, I. Yamazaki, Y. Tanimoto, Y. Hatano, M. Yamamato and H. Kono, J. Phys. Chem. 100 (1996) 19295.

[10] N. Ohta, H. Kawabata, S. Umeuchi and I. Yamazaki, Chem. Phys. Lett. 310 (1999) 397.

[11] R. Katoh, S. Sinha, S. Murata and M. Tachiya, J. Photochem. Photobiol. A 145 (2001) 23.

[12] H. Auweter, D. Ramer, B. Kunze and H. C. Wolf, Chem. Phys. Lett. 85 (1982) 325.

[13] H. Nishimura, A. Matsui and M. Iemura, J. Phys. Soc. Jpn. 51 (1982) 1341; H. Nishimura, T. Yamaoka, K. Mizuno, M. Iemura and A. Matsui, ibid. 53 (1984) 3999. 
[14] H. Tachikawa and L. R. Faulkner, Chem. Phys. Lett. 39 (1976) 436.

[15] Z. Salamon and H. Bassler, Chem. Phys. 100 (1985) 393.

[16] D. Weiss, R. Kietzmann, J. Mahrt, B. Tufts, W. Storck and F. Willing, J. Phys. Chem. 96 (1992) 5320.

[17] S. Akimoto, A. Ohmori and I. Yamazaki, J. Phys. Chem. B 101 (1997) 3753.

[18] N. Mataga, Y. Torihashi and Y. Ota, Chem. Phys. Lett. 1 (1967) 385.

[19] T. Iimori, A. M. Ara, T. Yoshizawa, T. Nakabayashi and N. Ohta, Chem. Phys. Lett. 402 (2005) 206.

[20] T. Nakabayashi, T. Morikawa and N. Ohta, Chem. Phys. Lett. 395 (2004) 346.

[21] E. A. Silinsh, G. A. Shlihta and A. J. Jurgis, Chem. Phys. 155 (1991) 389.

[22] W. Warta, R. Stehle and N. Karl, Appl. Phys. A 36 (1985) 163.

[23] H. Kasai, H. Kamatani, S. Okada, H. Oikawa, H. Matsuda and H. Nakanishi, Jpn. J. Appl. Phys. 35 (1996) L221.

[24] See as a review, W. Baumann, in Determination of Chemical Composition and Molecular Structure, Physical Methods of Chemistry-Part B, edited by B. W. Rossiter and J. F. Hamilton, Wiley 1989, Vol. 3, chap. 2, p. 45. 


\section{Figure captions}

Figure 1. Fluorescence spectrum, its first derivative spectrum and E-F spectrum of perylene doped in a PMMA film at $0.1 \mathrm{~mol} \%$ (from top to bottom) observed at $295 \mathrm{~K}$ (left) and at $70 \mathrm{~K}$ (right). The fieldstrength was $1.0 \mathrm{MV} / \mathrm{cm}^{-1}$, and the excitation wavelength was $413 \mathrm{~nm}$.

Figure 2. Fluorescence spectra (left) and E-F spectra (right) of perylene doped in a PMMA film at 1.0 mol\% observed at various temperatures in the range from 295 to $70 \mathrm{~K}$ (from top to bottom). The fieldstrength was $1.0 \mathrm{MVcm}^{-1}$, and the excitation wavelength was $397 \mathrm{~nm} . \mathrm{IF}_{\max }$ represents the maximum intensity of $\mathrm{I}_{\mathrm{F}}$.

Figure 3. Fluorescence spectra (left) and E-F spectra (right) of perylene doped in a PMMA film at 3.0 mol\% observed at various temperatures in the range from 295 to $70 \mathrm{~K}$ (from top to bottom). The fieldstrength was $1.0 \mathrm{MVcm}^{-1}$, and the excitation wavelength was $397 \mathrm{~nm}$. $\mathrm{IF}_{\max }$ represents the maximum intensity of $\mathrm{I}_{\mathrm{F}}$.

Figure 4. Thick solid line shows a simulation of the E-F spectra of perylene at $3 \mathrm{~mol} \%$ both at $295 \mathrm{~K}$ (left) and at $70 \mathrm{~K}$ (right) given by a linear combination of the fluorescence spectrum (broken line) and its first derivative spectrum (thin solid line). The E-F spectra observed at $1.0 \mathrm{MVcm}^{-1}$ are shown by a dotted line. 
Figure 5. ( $\bigcirc$ ) Plots of the fluorescence intensity, $\mathrm{I}_{\mathrm{F}}$, of perylene doped in a PMMA film at $1.0 \mathrm{~mol} \%$ in (a) and (b) and at $3.0 \mathrm{~mol} \%$ in (c) and ( $)$ its field-induced change $\left(\Delta \mathrm{I}_{\mathrm{F}}\right)$ relative to $\mathrm{I}_{\mathrm{F}}$ as a function of temperature. LE fluorescence at $448 \mathrm{~nm}$ and excimer fluorescence at $515 \mathrm{~nm}$ were monitored in (a) and (b), respectively, and excimer fluorescence at $530 \mathrm{~nm}$ was monitored in (c). The applied field strength was $1.0 \mathrm{MVCm}^{-1}$. 
$T=295 \mathrm{~K}$

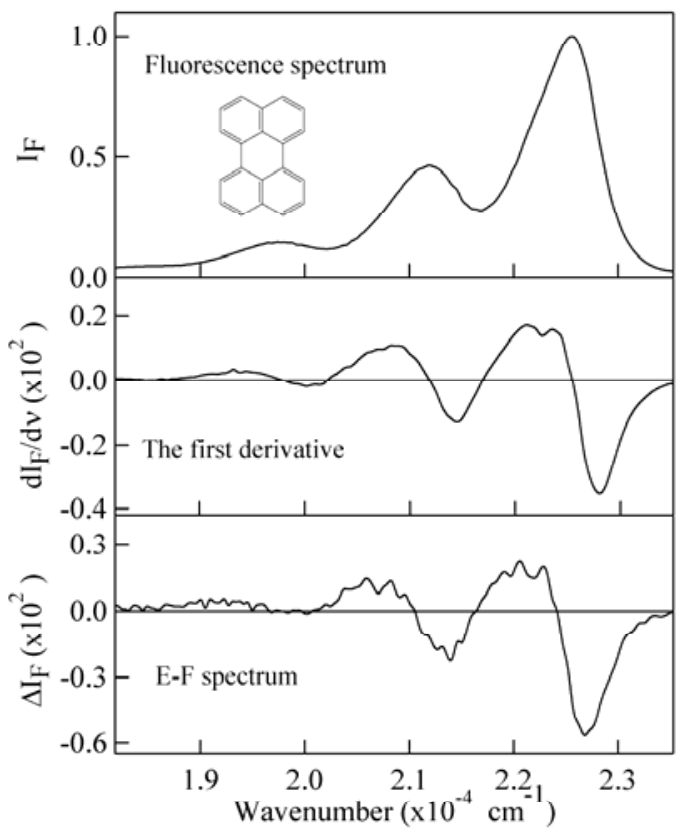

$T=70 \mathrm{~K}$

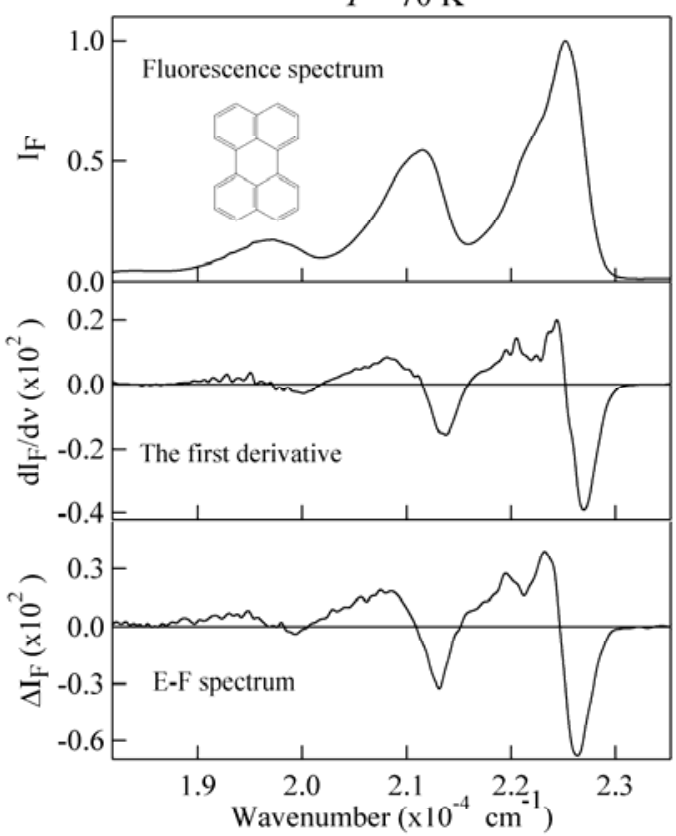

Fig. 1. A. M. Ara and N. Ohta et al. 
Fluorescence Spectra

\section{E-F Spectra}
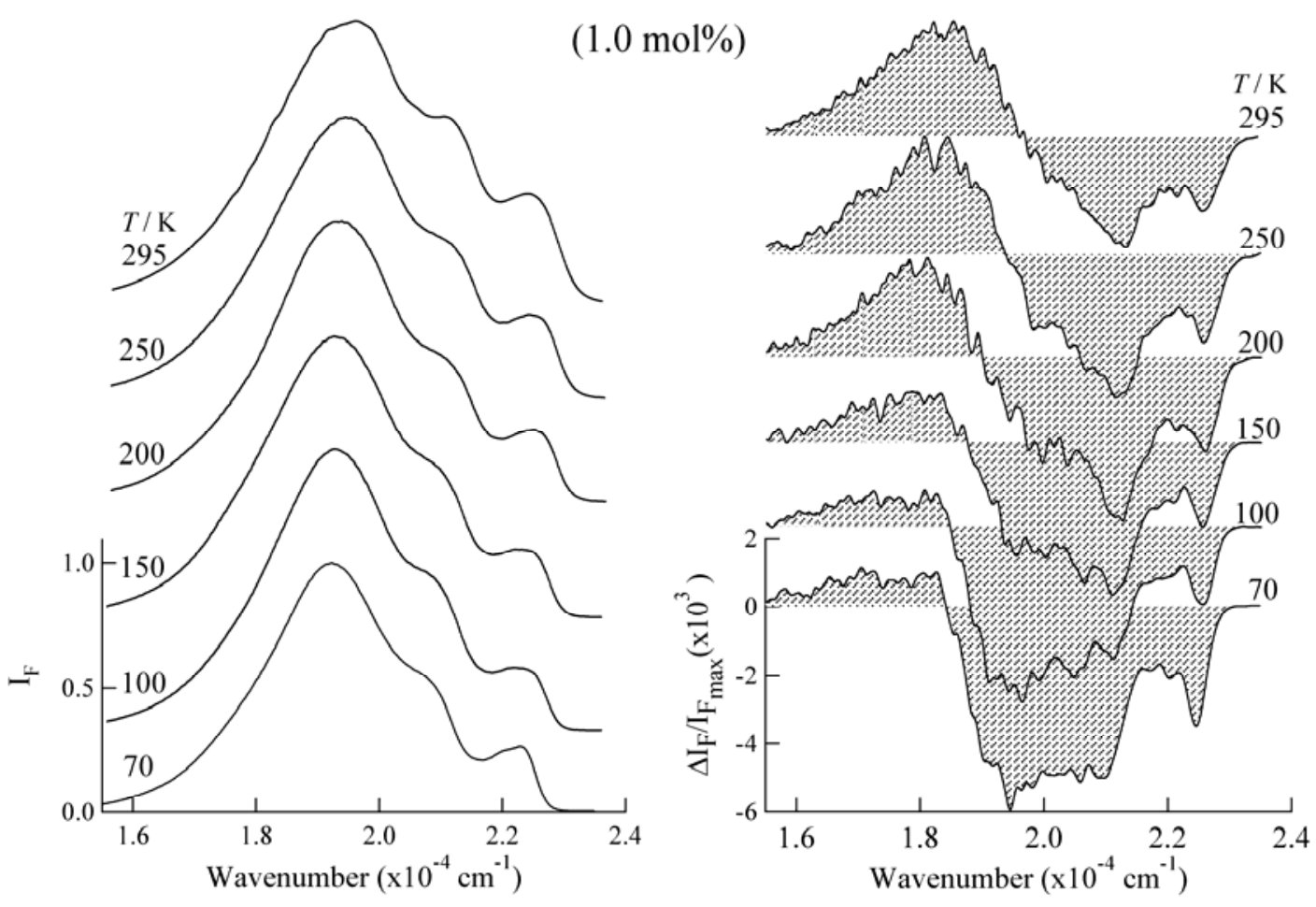

Fig. 2. A. M. Ara and N. Ohta et al. 
Fluorescence Spectra

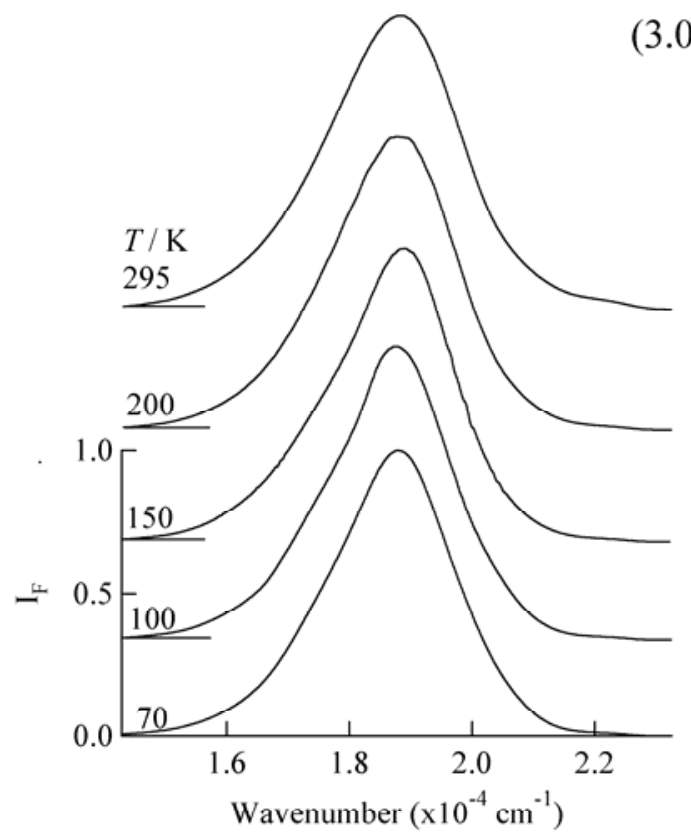

Fig. 3. A. M. Ara and N. Ohta et al.

\section{E-F Spectra}

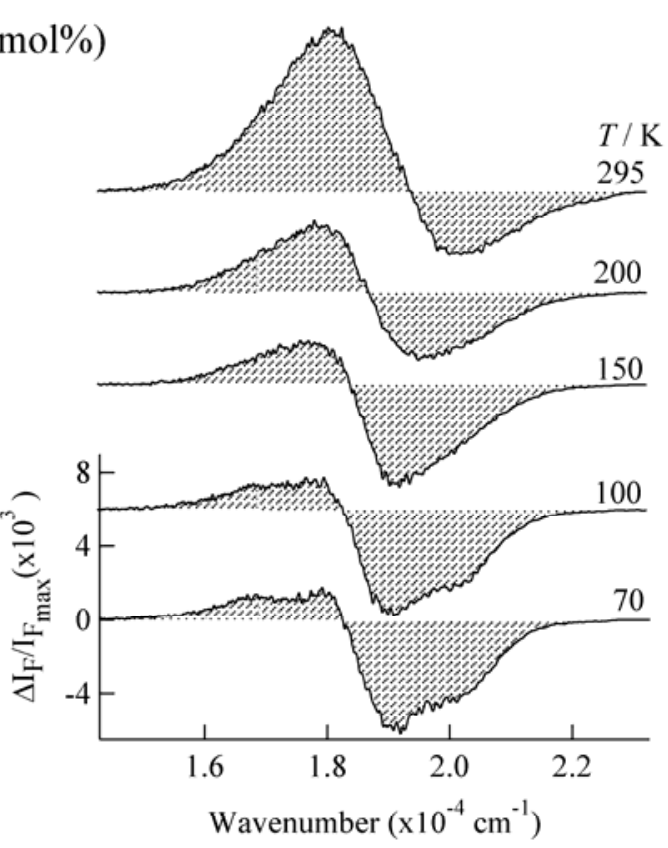



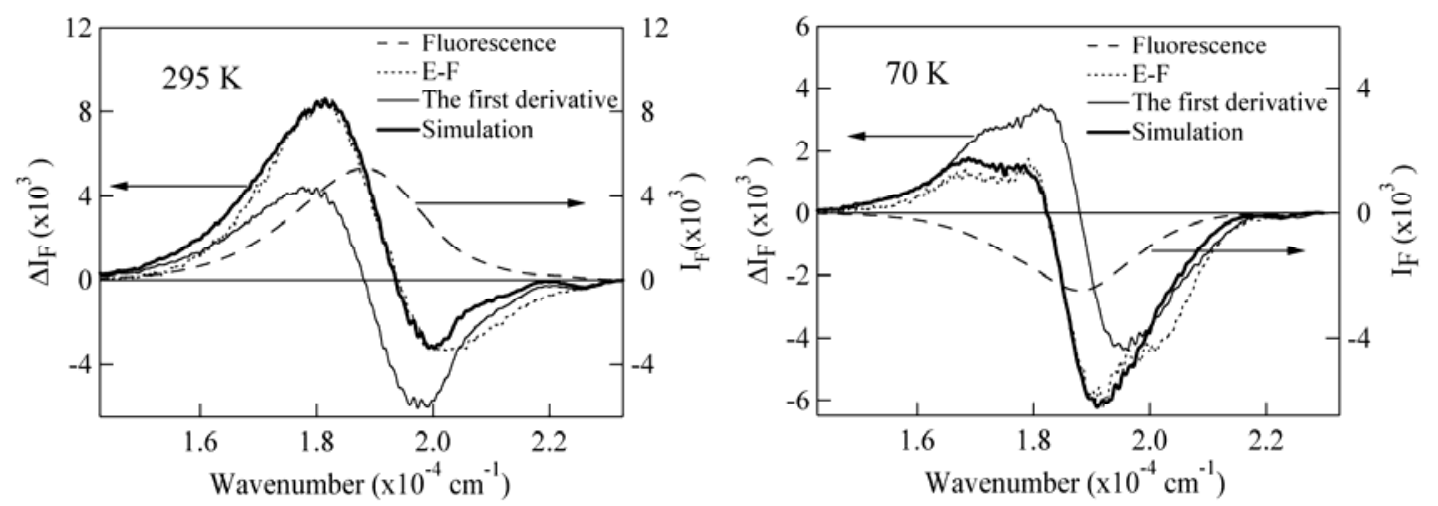

Fig. 4. A. M. Ara and N. Ohta et al 


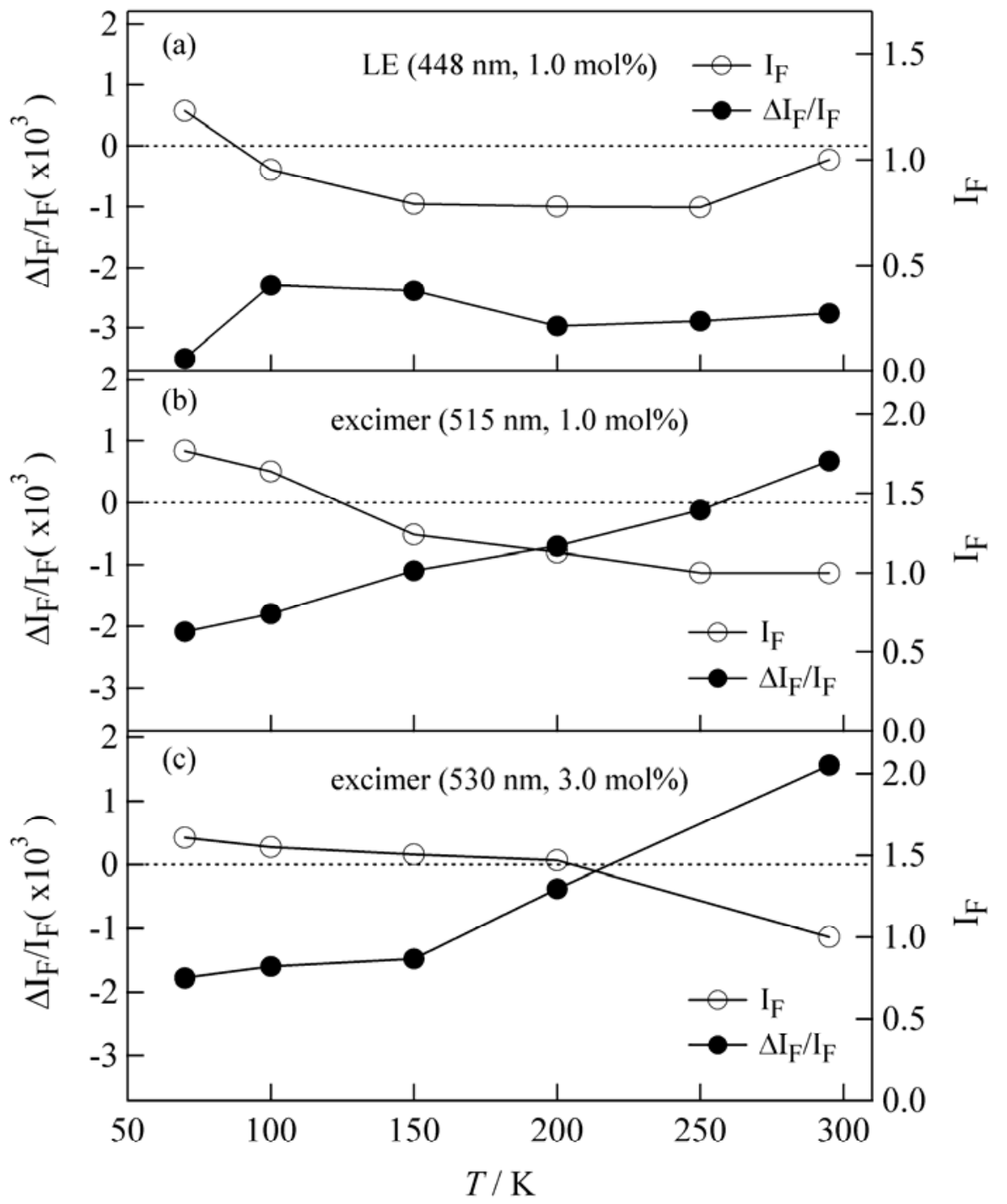

Fig. 5. A. M. Ara and N. Ohta et al. 